\title{
Mesenchymal Stem Cells and Articular Cartilage Repair: Clinical Studies and Future Direction
}

\author{
Shahid Punwar ${ }^{1}$ and Wasim S. Khan ${ }^{*}, 2$ \\ ${ }^{I}$ Department of Trauma and Orthopaedics, Frenchay Hospital, North Bristol NHS Trust, Bristol, BS16 1LE, UK \\ ${ }^{2}$ University College London Institute of Orthopaedics and Musculoskeletal Sciences, Royal National Orthopaedic \\ Hospital, Stanmore, Middlesex, HA7 4LP, UK
}

\begin{abstract}
Cartilage is frequently injured but shows little capacity for repair. Current treatment options include the use of procedures that stimulate repair through the stimulation of subchondral bone marrow and result in the formation of fibrocartilage. There is considerable interest in the use of cell-based treatment strategies and there are limited studies describing the use of mesenchymal stem cells for cartilage repair with promising early results. This paper reviews the current treatment strategies for articular cartilage, describes use of mesenchymal stem cells for articular cartilage repair along with the results of clinical studies, and describes the future direction that these strategies are likely to take.
\end{abstract}

Keywords: Cartilage repair, chondrogenesis, mesenchymal stem cells, osteoarthritis, tissue engineering.

Hyaline articular cartilage is a highly specialized tissue. It is characteristically avascular and depends on diffusion from cyclical loading during joint movement for nutrient acquisition [1]. As well as its shock absorbing function it enables synovial joints to articulate with extremely low frictional forces. Despite the best efforts of man to engineer smooth bearing surfaces, prosthetic joints cannot recreate anything like the low frictional environment of native synovial joints.

However articular cartilage does not readily repair itself following injury [2]. With pure chondral injuries the absence of a direct blood supply means that progenitor cells cannot reach the injured areas. Neither do surrounding chondrocytes migrate into cartilage defects and produce a reparative matrix. Repair relies on the mitotic potential of chondrocytes and a short lived increase in metabolic products, chiefly collagen and proteoglycans. However chondrocytes have low mitotic potential in vivo [3]. There is however some attempt at repair when cartilage damage breaches the underlying subchondral bone (osteochondral lesions). A primitive mesenchymal blood clot forms leading to the production of fibrocartilage [4]. This fibrocartilage has inferior biomechanical properties to hyaline cartilage and is the basis for marrow stimulation techniques such as microfracture.

Small cartilage defects can develop into widespread osteoarthritis, a particularly difficult problem to treat in the younger age group not suitable for fusion or joint replacement. Subsequently research interest into cartilage repair has been vast. A variety of methods have been developed in an attempt to repair articular cartilage defects. None are perfect. These include bone marrow stimulation techniques (subchondral drilling, abrasion, microfracture),

\footnotetext{
*Address correspondence to this author at the Department of Trauma and Orthopaedics, Frenchay Hospital, North Bristol NHS Trust, Bristol, BS16 1LE, UK; Tel: +44 (0) 7791 025554; Fax: + 44 (0) 7811 942192;

E-mail:wasimkhan@doctors.org.uk
}

osteochondral grafting (mosaicplasty) and autologous chondrocyte implantation (ACI). Recently autologous chondrocytes have been cultured on collagen membranes prior to re- implantation, a technique known as MACI (matrix assisted autologous chondrocyte implantation).

These procedures are limited to small and medium sized focal chondral and osteochondral defects. Mosaicplasty is limited by the need to create defects at donor sites, by poor healing between the grafts, and by difficulty in recreating the natural curvature of the joint. The ACI technique was first performed by Peterson et al. in 1994 [5] in Sweden. Although good results have been reported with ACI the quantity of harvested chondrocytes from non-weight bearing areas of cartilage is limited, and chondrocytes can dedifferentiate into fibroblasts when cultured in vivo. In addition harvested chondrocytes taken from already damaged joints may already be starting to show signs of degradation [6].

There is no evidence that the above techniques can reliably produce long lasting, durable articular cartilage.

An alternative, easily obtainable cell source with stable chondrocyte potential becomes necessary.

\section{ALTERNATIVE SOURCES OF CHONDROCYTES}

There are a number of chondroprogenitor cell pools within adult mammalian organisms that have the potential to differentiate into chondrocytes and form mature cartilage tissue. These include cells within the cambial layers of the perichondrium and periosteum, the synovial membrane, adipose tissue, muscle and the bone marrow stroma. Classically, stem cells are defined by their capacity to remain an undifferentiated state for a prolonged period while retaining the potential to differentiate along one lineage (unipotent), multiple lineages (multipotent), or into all three germ layers (pluripotent) [7]. 
Embryonic stem cells (ESCs) have been shown to differentiate into a variety of tissues, including cartilage [8]. They are harvested from the inner cell mass of the blastocyst and are pluripotent cells. However while ESCs have significant regeneration capacity, their clinical application has been limited due to several factors including concerns about teratoma formation, host immune rejection and the obvious ethical issues involved.

Adult stem cells have also been identified. They are categorized as either hemopoietic or nonhemopoietic depending on which cell lineages they have the potential to differentiate into. Nonhaemopoietic stem cells can give rise to cells of mesenchymal origin including bone, cartilage, muscle and adipose tissue. They are further characterized by an ability to adhere to plastic and the expression of specific surface antigens which allows their identification in vitro. These mesenchymal stem cells (MSCs) can be isolated from various tissues including blood, adipose, skin, mandible, trabecular bone and even the umbilical cord and placenta. Amongst the various sources, MSCs isolated from the bone marrow are considered to have the greatest potential for multilineage differentiation and have been the most characterized since their discovery in the early 1970 s by Friedenstein [9]. However harvesting of bone marrow is painful and not without the potential for complications. Hence an alternative easily obtainable source of cells with a small risk of complications would be ideal. Due to ease of harvest and abundance, adipose derived mesenchymal cells have become increasingly popular for use in many stem cell applications [10].

Another unique source of multipotent cells arises from Wharton's jelly in human umbilical cords [11].

Adult mesenchymal stem cells are easily obtainable in high numbers and expandable in vitro without losing their differentiation potential. Owing to their stem cell-like properties they are fairly well tolerated immunologically. Hence allogenic cells could be made readily available on a commercial basis avoiding the need for iliac crest bone marrow biopsies.

Although there have been some studies looking at direct application of these cells to cartilage defects most investigators have tried to use MSCs to engineer articular cartilage in vitro.

\section{CHONDROGENIC DIFFERENTIATION OF STEM CELLS}

It follows that the healing response seen in naturally ocurring full thickness cartilage defects is likely due to mesenchymal stem cells from the bone marrow entering the injured area via the blood vessels in the subchondral bone. However this repair is known to be incomplete and mainly composed of fibrocartilage [4]. Why is this? Caplan et al. [12] postulated that the number of progenitor cells in whole marrow aspirates was too low for effective cartilage production. They thus isolated and culture expanded MSCs from marrow aspirates before transplantation, with much better results in animal models.

It is also important to remember that hyaline cartilage is formed of several different cell layers. The uppermost layer is formed by articular chondrocytes progressing to hypertrophic chondrocytes and underlying subchondral bone. Caplan et al. [12] in a rabbit model observed that mesenchymal stem cells transplanted into full thickness cartilage defects could recreate this layered arrangement. Detailed analysis showed that the implanted cells followed the embryonic sequence. Initially the cells rapidly differentiated into embryonic cartilage. Chondrocytes at the defect base then differentiate into hypertrophic chondrocytes which are subsequently replaced by vascalature and host osteoblasts leading to the production of new subchondral bone. The top cartilage remained as articular cartilage probably from exposure to synovial factors.

\section{TISSUE ENGINEERED APPROACHES CARTILAGE REPAIR}

TO

Tissue engineering can be defined as the art of reconstituting mammalian tissues, both structurally and functionally [13]. The process can be conducted either entirely or partly in vitro with the engineered tissues then transplanted into articular cartilage defects. There are essentially four main components required for successful tissue engineering. Firstly a physical scaffold to provide an adequate three-dimensional surrounding for handling. Secondly, appropriate cells which are able to differentiate and maintain the specific cell phenotype. Thirdly, the addition of the right bioactive substances such as growth factors, cytokines or hormones as a suitable stimulus for specific lineage differentiation of the cells. Finally the tissues need to be able to withstand the mechanical and biochemical environment into which they are placed.

Specifically related to repair of articular cartilage repair an available cell population is needed which can meet the metabolic demands of restoring a functional extracellular matrix. This can be accomplished with either fully differentiated chondrocytes, mesenchymal stem cells, or gene transduced cells. As well as an appropriate scaffold to fill the void of the defect a method is also needed for adequate internal fixation of the implant or cell based graft within the synovial environment. Fixation must be able to withstand the forces of joint motion but must not interfere with the repair process.

Using these principles autologous bone marrow stromal cells have been used for the repair of full-thickness articular cartilage defects in humans, resulting in stable fibrocartilage tissue formation at the defect site [14, 15]. However it is likely that in order to repair cartilage defects with a stable hyaline-rich tissue the delivery of factors stimulating chondrogenesis and maintenance of the articular cartilage phenotype needs to be more efficient than purely exogenous administration.

\section{GENE-ENHANCED TISSUE ENGINEERING}

The application of gene transfer to articular cartilage was pioneered by Evans and co-workers as a means to treat arthritis [16]. Essentially these techniques aim to program cells to produce bioactive factors to promote their specialized functions. An example would be isolating the gene for bone morphogenetic protein (BMP) and introducing it into primary periosteal cells, thereby encouraging the osteogenic and chondrogenic capabilities of the cells. Therapeutic genes can be delivered to the synovium or 
directly to the cartilage lesion. Gene transfer to cartilage defects can be achieved by either direct vector administration to cells located at or surrounding the defects, or by transplantation of genetically modified chondrogenic cells into the defect [17].

\section{CLINICAL EXPERIENCE WITH MESENCHYMAL STEM CELLS}

Mesenchymal stem cells of bone-marrow stromal origin have been used in an attempt to produce articular cartilage in several animal models and compared with other techniques [18]. Cells have been combined with different matrices, such as Type I collagen gels or modified hyaluronan and different growth factors. Transforming growth factor- $\beta$ (TGF- $\beta$ ), BMP-2 and insulin growth factor-1 (IGF-1) are emerging among the most popular candidates shown to encourage chondrogenic differentiation of MSCs. There are limited human in vivo studies at present. Comparison between studies is difficult as no single protocol has yet emerged as the best model for cartilage engineering from stem cells. Both two-step and one-step techniques have been developed as well as percutaneous methods of cell application.

Kuroda et al. 2007 [14] treated a large full thickness articular cartilage defect in the femoral condyle of an athlete with autologous bone-marrow stromal cells. Bone marrow was aspirated from the patient's iliac crest 4 weeks before surgery and the cells were expanded in culture after removing the erythrocytes. Cells were then embedded within a collagen gel which was transferred to the articular cartilage defect and covered with an autologous periosteal flap. Seven months after surgery, arthroscopy revealed the defect to be covered with smooth tissues. Histologically the defect was covered with a hyaline-like type of cartilage tissue. One year after surgery, the clinical symptoms had improved significantly and the patient had returned to his previous activity level.

Gianinni et al. [19] have developed a one-step repair technique with bone-marrow-derived cells. They have reported good clinical results in both the ankle and knee [19, 20]. As well as bone marrow aspiration, the technique involves creating a platelet-rich fibrin gel from a sample of the patient's own blood. The bone marrow is concentrated by centrifugation and implanted onto a hyaluronic acid membrane which is then placed surgically into the cartilage defects and covered with the platelet-rich gel. Wakitani et al. [21] reported on three knee cases using undifferentiated bone marrow derived MSC associated with a collagen sheet to repair chondral defects. They showed an improvement in IKDC (International Knee Documentation Committee) however in two patients they identified a fibrocartilage like tissue in the defect similar to their previous study [14]. This suggests that autologous stem cells do not always differentiate into mature hyaline cartilage. Haleem et al. [22] have used MSCs transplanted on a scaffold of platelet-rich fibrin glue (PR-FG) in the treatment of articular cartilage defects in the knees of 5 patients. Symptoms and knee scores improved in all patients. Platelet-rich fibrin glue may be an ideal MSC scaffold as the $\alpha$-secretory granules of platelets contain both TGF- $\beta 1$ and IGF-1 [23-26].
Overall the results achieved using bone-marrow stromal cells have been similar to those yielded using other cellmatrix systems, the repair tissue formed being principally fibrocartilaginous in nature, but of variable quality and durability [4].

\section{FUTURE DIRECTIONS}

In order to engineer cartilage with stem cells researchers must control many factors, including differentiation and cartilage matrix synthesis, particularly collage II production. Because cartilage is a primarily avascular tissue a relatively hypoxic environment is created. It has been postulated that this hypoxia is important for chondrogenesis and consequently in vitro engineering efforts have attempted to mimic this environment generally showing enhancement of chondrogenesis. Hypoxic conditions have been shown to significantly alter the chondrogenic differentiation process whereby cells cultured in these conditions had an enhanced ability to produce collagen type II and glycosaminoglycans. This resulted in better biomechanical functionality in artificial cell constructs [29].

We have previously showed that bone marrow derived MSCs undergoing chondrogenic differentiation exhibit a greatly enhanced induction of chondrogenesis under hypoxic conditions (5\% oxygen) as compared to normoxic culture conditions ( $20 \%$ oxygen). Changes in gene expression suggesting enhanced chondrogenesis were apparent in lower oxygen conditions, and there was greater staining for collagen type II (Fig. 1) [30]. A similarly enhanced chondrogenic response was seen when we cultured infrapatellar fat pad (IPFP) derived stem cells under conditions of low oxygen tension (Fig. 2) [31]. We have also showed that chondrogenic differentiation is potentiated by FGF-2 supplemented expansion of infrapatellar fat pad (IPFP) derived cells. The effect of FGF-2 expansion was quantified by a greater increase in collagen type II expression, as compared to non-FGF-2-expanded cells, and this was confirmed on immunohistochamistry. The IPFP cells were shown to have capacity for multidifferentiation by $3 \mathrm{G} 5$ staining for pericytes, which are known to have such a potential [32].

However the actual differentiation of mesenchymal stem cells into chondrocytes may not be of paramount importance. MSCs have been proposed as a natural system for tissue repair [27]. They have been shown to seemingly enhance the repair of damaged tissue when applied exogenously in a variety of experimental models. However in the vast majority of these studies the therapeutic efficacy did not correlate with the efficiency of engraftment, which was in general low. These findings suggest that the ability to repair was very likely secondary not only to the differentiation of the cells into the appropriate phenotype, but by the secretion of soluble factors that altered the tissue microenvironment. In other words stem cells may have a trophic effect on injured tissues. Research has shown that MSCs in vitro produce a variety of factors that influence a broad range of biological functions, including angiogenesis, and also secrete neuroregulatory peptides and cytokines with critical roles in inflammation and repair [28]. 


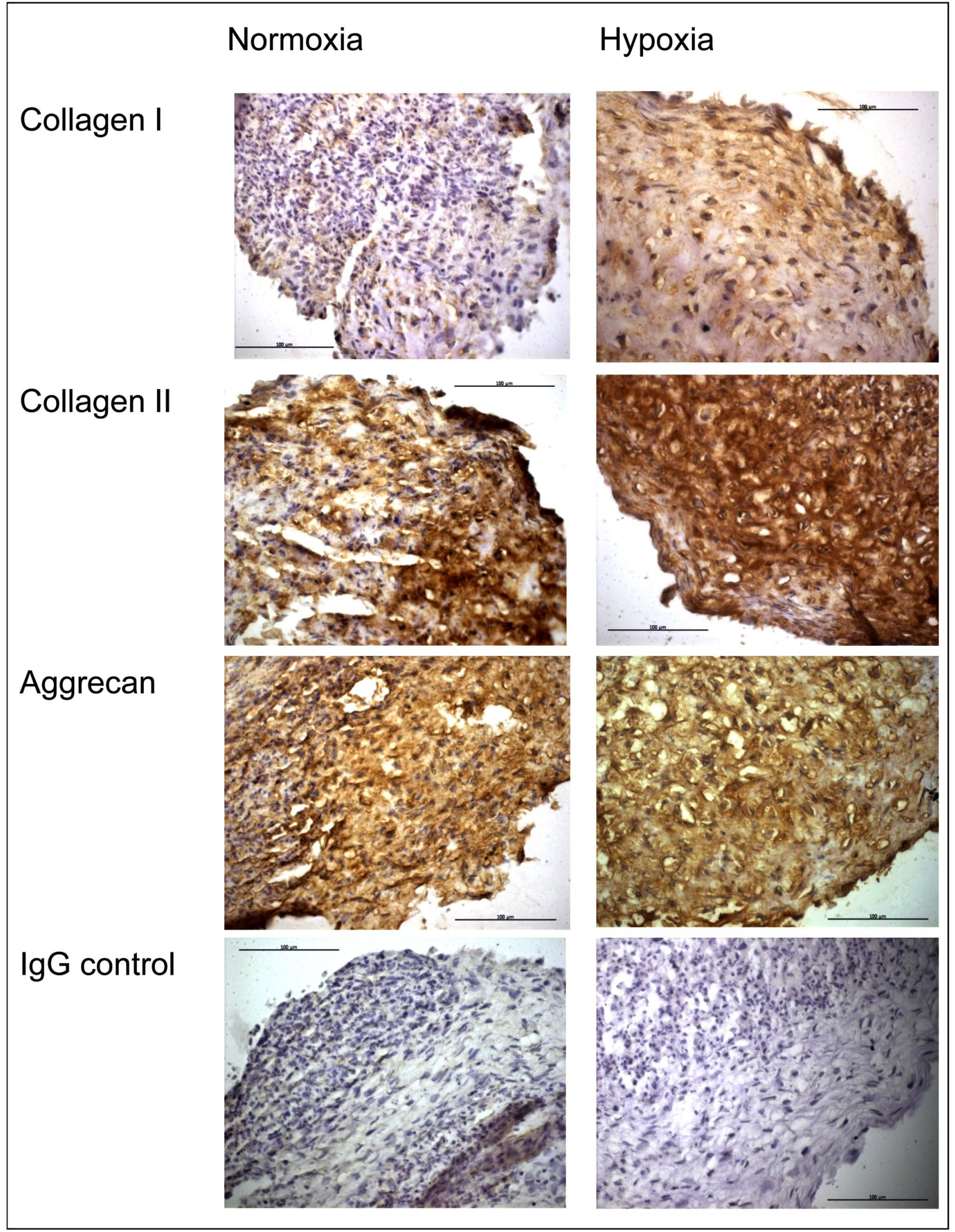

Fig. (1). Immunohistochemistry of chondrogenic cultures of bone marrow derived MSCs. Immunohistochemical staining for collagen type I and II, aggrecan and control IgG in cell aggregates following chondrogenic differentiation for 14 days under normoxic and hypoxic culture conditions [30].

Ultimately large scale clinical trials are needed to evaluate the effectiveness and safety of MSC therapy in the treatment of cartilage defects. Over a 100 clinical trials utilizing exogenous bone marrow derived stem cells to treat a wide variety of conditions are registered with ClinicalTrials.gov [33, 34]. Although many are investigating stem cell use in orthopaedic associated tissue regeneration, limited evidence is currently available to support routine use. 
Centeno et al. [29] have injected bone marrow-derived stem cells percutaneously into the knee of a patient with radiographic signs of degenerative joint disease. Six months later they observed a significant cartilage growth and pain was improved. These findings may support the injection of MSCs directly into joints against filling up cartilage defects with complex engineered scaffolds.

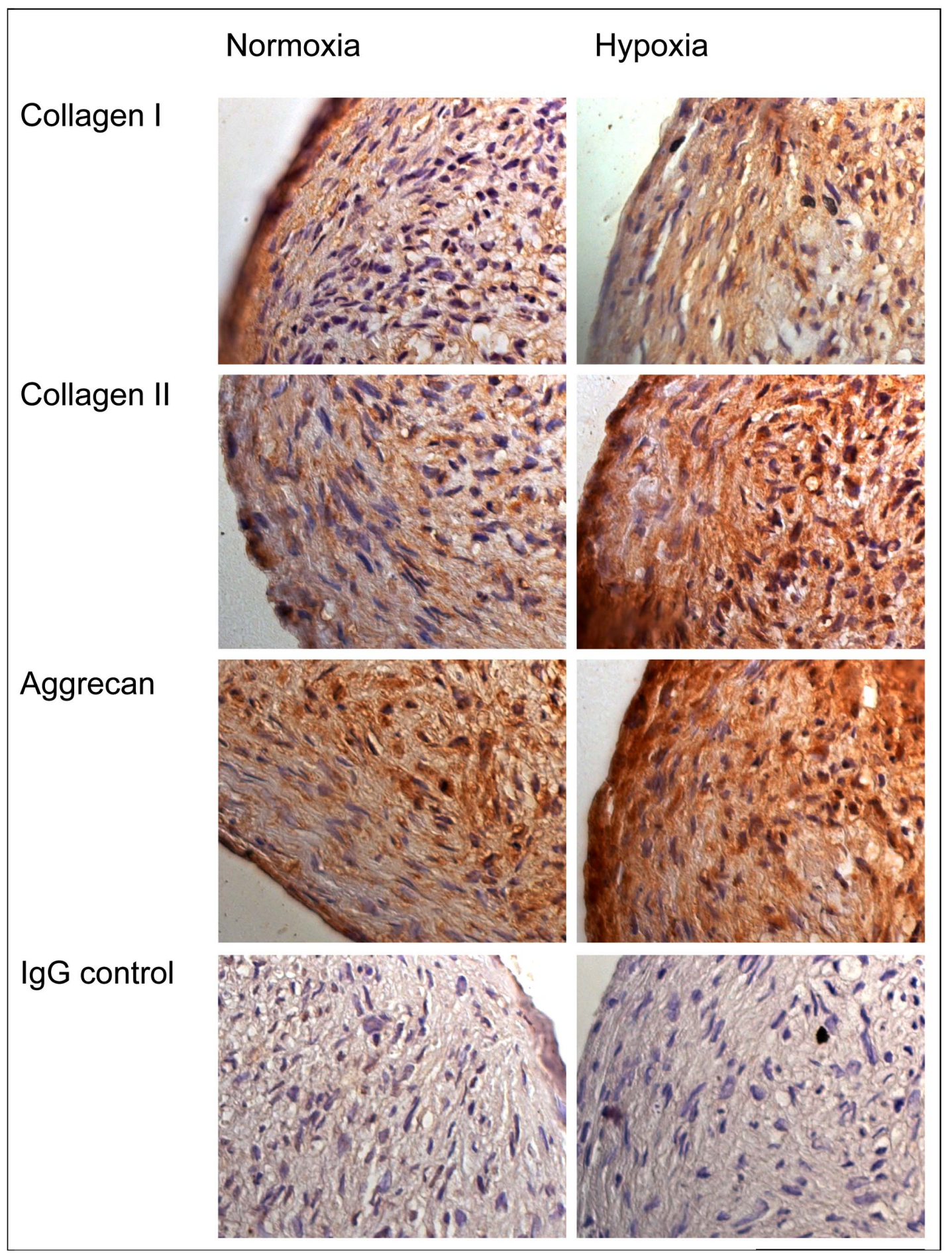

Fig. (2). Immunohistochemical staining in chondrogenic cultures of infrapatellar fat pad derived MSCs. Immunohistochemical staining for collagen type I and II, aggrecan and control IgG in cell aggregates following chondrogenic differentiation for 14 days under normoxic and hypoxic culture conditions [31].

There is no doubt that mesenchymal stem cells are readily available from a number of sources and they clearly have the ability to regenerate damaged mammalian tissues. However further research is needed to elucidate exactly how they exert their reparative actions. It may be that a revolutionary new field of MSC medicine will emerge, augmenting the normal healing process of injured tissues [35]. The potential therapeutic possibilities appear endless. 


\section{ACKNOWLEDGEMENT}

None declared.

\section{CONFLICT OF INTEREST}

None declared.

\section{REFERENCES}

[1] O'Hara BP, Urban JPG, Maroudas A. Influence of cyclic loading on the nutrition of articular cartilage. Ann Rheum Dis 1990; 49(7): 536-39.

[2] Buckwalter JA, Mankin HJ. Articular cartilage repair and transplantation. Arthritis Rheum 1998; 41: 1331-42.

[3] Brittberg M, Lindahl A, Nilsson A, et al. Treatment of deep cartilage defects in the knee with autologous chondrocyte transplantation. N Engl J Med 1994; 331: 889-95.

[4] Hunziker E. Articular cartilage repair: basic science and clinical progress. A review of the current status and prospects. Osteoarthritis Cartilage 2001; 10: 432-63.

[5] Peterson L, Minas T, Brittberg $\mathrm{M}$, et al. Treatment of osteochondritis dissecans of the knee with autologous chondrocyte transplantation: results at two to ten years. J Bone Joint Surg Am 2003; 85A(Suppl 2): 17-24.

[6] Tew SR, Clegg PD, Brew CJ, Redmond CM, Hardingham TE. SOX9 transduction of a human chondrocytic cell line identifies novel genes regulated in primary human chondrocytes and in osteoarthritis. Arthritis Res Ther 2007; 9: R107.

[7] Young HE. Existence of reserve quiescent stem cells in adults, from amphibians to humans. Curr Top Microbiol Immunol 2003; 280: 71-109.

[8] Thompson JA. Embryonic stem cell lines derived from human blastocysts. Science 1998; 5391: 1145-47.

[9] Friedenstein A, Kuralesova A. Osteogenic precursor cells of bone marrow in radiation chimeras. Transplantation 1971; 12(2): 99-108.

[10] Guilak F, Estes B, Diekman B, et al. Nicolas Andry Award: multipotent adult stem cells from adipose tissue for musculoskeletal tissue engineering. Clin Orthop Relat Res 2010; 468: 2530-40.

[11] Sarugaser R, Lickorish D, Baksh D, et al. Human umbilical cord perivascular (HUCPV) cells. A source of mesenchymal progenitors. Stem Cells 2005; 23: 220-29.

[12] Caplan A, Elyaderani M, Mochizuki et al. Principles of cartilage repair and regeneration. Clin Orthop Relat Res 1997; 342: 254-69.

[13] Czaki C, Schneider P, Shakibaei M. Mesenchymal stem cells as a potential pool for cartilage tissue engineering. Ann Anat 2008; 190: 395-412.

[14] Kuroda R, Ishida K, Matsumoto T, et al. Treatment of a fullthickness articular cartilage defect in the femoral condyle of an athlete with autologous bone-marrow stromal cells. Osteoarthritis Cartilage 2007; 15: 226-31.

[15] Wakitani S, Mitsuoka T, Nakamura N, et al. Autologous bone marrow stromal cell transplantation for repair of full-thickness articular cartilage defects in human patellae: two case reports. Cell Transplant 2004; 13(5): 595-600.

[16] Evans CH, Gouze JN, Gouze E, et al. Osteoarthritis gene therapy. Gene Ther 2004; 11(4): 379-89.

[17] Steinert A, Noth U, Tuan R. Concepts in gene therapy for cartilage repair. Injury 2008; 39(Suppl 1): S97-113.

[18] Hui Jh, Chen F, Thambyah A, Lee EH. Treatment of chondral lesions in advanced osteochondritis dissecans: a comparative study of the efficacy of chondrocytes, mesenchymal stem cells, periosteal graft and mosaicplasty (osteochondral autograft) in animal models. J Pediatr Orthop 2004; 24(4): 427-33.

[19] Giannini S, Buda R, Vannini F, et al. One-step bone marrowderived cell transplantation in talar osteochondral lesions. Clin Orthop Relat Res 2009; 467: 3307-20.

[20] Buda R, Vannini F, Cavallo M, et al. Osteochondral lesions of the knee: a new one-step repair technique with bone-marrow derived cells. J Bone Joint Surg Am 2010; 92 (Suppl 2): 2-11.

[21] Wakitani S, Nawata M, Tensho K, Okabe T, Machida H, Ohgushi H. Repair of articular cartilage defects in the patella-femoral joint with autologous bone marrow mesenchymal cell transplantation: three case reports involving nine defects in five knees. J Tissue Eng Regen Med 2007; 1: 74-9.

[22] Haleem A, El-Singergy A, Sabry D, et al. The clinical use of human culture-expanded autologous bone marrow mesenchymal stem cells transplanted on platelet-rich fibrin glue in the treatment of articular cartilage defects: a pilot study and preliminary results. Cartilage 2010; 1(4): 253-61.

[23] Mankin HJ, Jennings LC, Treadwell BV, Trippel SB. Growth factors and articular cartilage. J Rheumatol Suppl 1991; 27: 66-67.

[24] Nair MB, Varma HK, John A. Platelet-rich plasma and fibrin gluecoated bioactive ceramics enhance growth and differentiation of goat bone marrow-derived stem cells. Tissue eng Part A 2009; 15(7): 1619-31.

[25] Phornphutkul C, Wu KY, Yang X, Chen Q, Grupposo PA. Insulinlike growth factor-I signalling is modified during chondrocytes differentiation. J Endocrinol 2004; 183(3): 477-86.

[26] Schmidt MB, Chen EH, Lynch SE. A review of the effects of insulin-like growth factor and platelet derived growth factor on in vivo cartilage healing and repair. Osteoarthritis Cartilage 2006; 14(5): 403-12.

[27] Phinney DG, Prockop DJ. Concise review: mesenchymal stem/multipotent stromal cells: the state of transdifferentiation and modes of tissue repair - current views. Stem Cells 2007; 25(11): 2896-902.

[28] Caplan AI, Dennis JE. Mesenchymal stem cells as trophic mediators. J Cell Biochem 2006; 98: 1076-84.

[29] Centeno CJ, Busse D, Kisiday J, Keohan C, Freeman M, Karli D. Increased knee cartilage volume in degenerative joint disease using percutaneously implanted, autologous mesenchymal stem cells. Pain Physician 2008; 11: 343-53.

[30] Khan WS, Adesida AB, Tew SR, Lowe ET, Hardingham TE. Bone marrow-derived mesenchymal stem cells express the pericyte marker $3 \mathrm{G} 5$ in culture and show enhanced chondrogenesis in hypoxic conditions. J Orthop Res 2010; 28(6): 834-40.

[31] Khan WS, Tew SR, Adesida AB, Hardingham TE. Human infrapatellar fat pad-derived stem cells express the pericyte marker 3G5 and show enhanced chondrogenesis after expansion in fibroblast growth factor-2. Arthritis Res Ther 2008; 10(4): R74.

[32] Khan WS, Adesida AB, Hardingham TE. Hypoxic conditions increase hypoxia-inducible transcription factor 2alpha and enhance chondrogenesis in stem cells from the infrapatellar fat pad of osteoarthritis patients. Arthritis Res Ther 2007; 9(3): R55.

[33] Koay EJ, Athanasiou KA. Hypoxic chondrogenic differentiation of human embryonic stem cells enhances cartilage protein synthesis and biomechanical functionality. Osteoarthritis Cartilage 2008; 16 : 1450-56.

[34] Shenaq D, Rastegar F, Petkovic D, et al. Mesenchymal progenitor cells and their orthopaedic applications: forging a path towards clinical trials. Stem Cells Int 2010; 519028.

[35] Caplan AI. Mesenchymal stem cells: the past, the present, the future. Cartilage 2010; 1(1): 6-9. 